\title{
Intravesical Protrusion of the Prostate as a Predictive Method of Bladder Outlet Obstruction
}

\author{
Leonardo O. Reis, Guilherme C. Barreiro, Jamal Baracat, Alessandro Prudente, Carlos A. \\ D'Ancona
}

Division of Urology, School of Medicine, University of Campinas, Campinas, Sao Paulo, Brazil

\begin{abstract}
Objective: Pressure-flow study is the gold standard for diagnosis of bladder outlet obstruction (BOO). A prospective study was carried out to compare urodynamic evaluation and measurement of intravesical protrusion of the prostate for diagnosing BOO.

Materials and Methods: Patients presenting with lower urinary tract symptoms (LUTS) associated with benign prostatic hyperplasia and suspected BOO were prospectively evaluated through conventional urodynamics and classified according to the bladder outlet obstruction index (BOOI). They also underwent abdominal ultrasound measurement of the intravesical prostatic protrusion (IPP) and prostatic volume. The IPP was classified into three stages: grade I under 5 mm; grade II, between 5 and $10 \mathrm{~mm}$; and grade III over $10 \mathrm{~mm}$.

Results: Forty-two patients, mean age $64.8 \pm 8.5$ years were enrolled. Transabdominal ultrasound determined a mean prostatic volume of $45 \pm 3.2 \mathrm{~mL}$. Achieved IPP's values were the following: grade I - 12 (28.5\%), grade II - 5 - (12\%) and grade III - $25(59.5 \%)$. The results of prostate volume differed significantly between obstructed and non-obstructed men $(\mathrm{p}=0.033)$ and for IPP among obstructed, inconclusive and non-obstructed men $(\mathrm{p}=0.016)$. For IPP, the area under ROC curve was 0.758 (95\% confidence interval - 0.601 to 0.876$)$, and the cutoff point to indicate BOO was $5 \mathrm{~mm}$ with $95 \%$ sensitivity (75.1 - 99.2) and $50 \%$ specificity (28.2 - 71.8).

Conclusion: IPP and prostatic volume measured through abdominal ultrasound are noninvasive and accessible methods that significantly correlate to urinary BOO, and are useful in the diagnosis of male urinary obstructive problems.
\end{abstract}

Key words: bladder outlet obstruction; prostate; volume; flowmetry; sensitivity and specificity

Int Braz J Urol. 2008; 34: 627-37

\section{INTRODUCTION}

Bladder outlet obstruction (BOO) is characterized by increased detrusor pressure and reduced urinary flow rate. Pressure-flow studies are the gold standard for BOO determination. However, this method is an invasive and expensive procedure with limited availability.. Therefore, attempts have been made to diagnose $\mathrm{BOO}$ through noninvasive methods that can be divided into 2 categories: non-urodynamically based measurements and noninvasive urodynamics.
Non-urodynamically based measurements include symptoms, post-void residual urine (PVR), Prostate Specific Antigen (PSA) and ultrasound derived measurements, such as prostate volume, bladder wall thickness, bladder weight and intravesical prostatic protrusion (IPP). Noninvasive urodynamics include uroflowmetry, use of a penile cuff, the condom-method and Doppler urodynamics (1).

It is well known that the prostate's anatomic conformation together with intravesical prostatic protrusion (IPP) may affect normal voiding. 
Earlier studies have previously demonstrated that the ultrasonographic measurement of IPP could identify BOO. A total of 200 patients were assessed with invasive urodynamics and transabdominal ultrasound. The relationship of IPP to BOO showed that as IPP grade increased in severity, BOO grade also increased. The sensitivity and specificity of diagnosing BOO were $76 \%$ and $92 \%$ for over $10 \mathrm{~mm} \mathrm{IPP,} 17 \%$ and $53 \%$ for between 5 and $10 \mathrm{~mm}$ IPP and $7 \%$ and $56 \%$ for under $5 \mathrm{~mm} \mathrm{IPP}$, respectively. PVR more than $100 \mathrm{~mL}$ showed $75 \%$ sensitivity and $91 \%$ specificity for predicting $\mathrm{BOO}$ in the population studied (2).

The objective of this study was to define how the IPP and prostate volume, measured through abdominal ultrasound, might alter voiding and determine the accuracy of this measurement compared to conventional urodynamics in diagnosing BOO.

\section{MATERIALS AND METHODS}

A prospective study was carried out in LatinAmerican patients presenting with lower urinary tract symptoms (LUTS) and evaluated by urinalysis to exclude urinary tract infection.

Patients who had been previously submitted to urologic surgeries, or had urologic neoplasia, bladder calculus or presented any type of neurological abnormality or using alpha-blockers, anticholinergics, antiandrogens or another medications which may affected the voiding patterns were excluded from this study.

In the period ranging from June to August/2005, after Ethics Committee approval and written informed consent, these patients were evaluated using anamnesis, International Prostatic Symptoms Score (IPSS) and IPSS Quality of Life (IPSS-QoL) questionnaires, physical, neurological, digital rectal examination and conventional urodynamic evaluation (Dynapack, Dynamed, 2004) and classified according to BOOI.

Urodynamics were done according to the "good urodynamic practices" recommended by the International Continence Society (3). Bladder outlet obstruction index (BOOI), defined as the detrusor's pressure at the maximum urinary flow (pdet_qmax) minus two times the maximum flow (qmax): $\overline{\mathrm{BOOI}}=$ pdet_qmax - 2 x qmax. Values below 20 were considered non-obstructed, between 20 and 40 inconclusive and higher than 40, obstructed (4). Postvoid residual urine volume was measured during urodynamic investigation, after free uroflowmetry (free flow).

After one week the patients underwent an ultrasound study performed by the same physician (J.B.) blinded to the urodynamic results performed by L.O.R. Abdominal ultrasound (Toshiba model Powervision 6000) was performed in the sagittal plane, using transductor frequencies between 3 and $6 \mathrm{MHz}$, and IPP along with the prostatic volume were measured. According to Yuen et al. (5), the bladder was filled with at least $100 \mathrm{~mL}$ of urine in order to consider the IPP determination; this was achieved through the ingestion of one liter of water in a two hour period after voiding. IPP was defined by the distance from the tip of the prostate's protrusion into the vesical lumen to the bladder neck measured in millimeters (Figure-1). Measurements were divided into three stages: grade I $<5 \mathrm{~mm}$, grade II $5-10 \mathrm{~mm}$, grade III $>10 \mathrm{~mm}$ (2). Prostate volumes were determined through software (Powervision 600) for automatic measurement and expressed in milliliters $(\mathrm{mL})$.

The statistical analysis was performed through Kruskal-Wallis and Dunn's post test to multiple comparison, and area under ROC curve, using MedCalc version 5.00.019 and SAS System for Windows version 9.1.3.

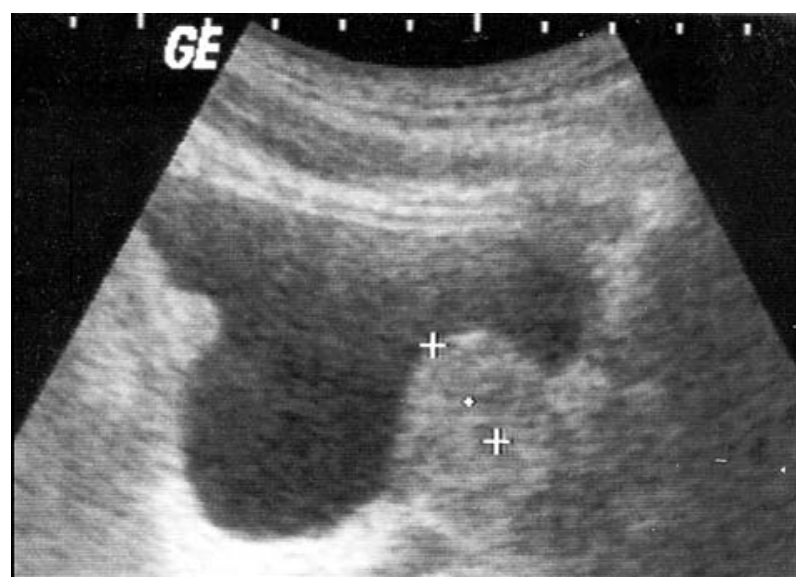

Figure 1 - Sagittal views of bladder and prostate using transabdominal ultrasonography. Vertical distance from tip of protrusion to base of bladder is the intravesical prostatic protrusion measurement. 
Table 1 - Clinical and demographic characteristics.

\begin{tabular}{lc}
\hline Clinical and Demographics Characteristics & Rate \\
\hline Age & $64.9(56$ to 73$)$ years \\
Latin-American Caucasians & $100 \%$ \\
IPSS & $13(6$ to 20$)$ \\
Ultrasound transabdominal examination & $45(5.5$ to 155$) \mathrm{mL}$ \\
Qmax & $8.5(5.5$ to 13$) \mathrm{mL} / \mathrm{s}$ \\
Pdet_Qmax & $58.1(35$ to 126$)$ \\
Post voiding residue & $70(0$ to 250$) \mathrm{mL}$ \\
\hline
\end{tabular}

IPPS = International Prostate Symptom Score.

\section{RESULTS}

The clinical and demographic characteristics are shown in Table-1.

The pressure/flow study showed mean Qmax of $8.5 \pm 4.3 \mathrm{~mL} / \mathrm{s}$, Pdet.qmax of $58.1 \pm 26 \mathrm{~cm} \mathrm{H}_{2} \mathrm{O}$, and postvoid residual urine volume after free flow of $70 \pm 177 \mathrm{~mL}$. Based on BOOI, 20 (47.6\%) patients presented obstruction, $12(28.5 \%)$ were inconclusive and $10(23.9 \%)$ did not present obstruction. The mean BOOI was 28.6 (SD 13.4).

IPP's values obtained were as follows: grade I - $12(28.5 \%)$, grade II - 5 - (12\%) and grade III - 25 $(59.5 \%)$.

Comparing prostatic volume and IPP with BOOI we found according to Kruskal-Wallis and Dunn's post test that the results of prostate volume differed significantly between obstructed and nonobstructed men $(p=0.033)$ and the results of IPP differed significantly among obstructed, inconclusive and nonobstructed men $(\mathrm{p}=0.016)$, Table- 2 .

Table-3 demonstrates that IPP's grade III reached up to $80 \%$ sensitivity and $68 \%$ specificity for diagnosing BOO. Positive predictive value was $70 \%$ and the negative predictive value $79 \%$.

Considering the slight IPP superiority over prostate volume to detect obstruction, we calculated the IPP cutoff point to indicate obstruction as $5 \mathrm{~mm}$ with $95 \%$ sensitivity $(75.1-99.2)$ and $50 \%$ specificity $(28.2-71.8)$; likelihood ratio of positive test result 1.90 and likelihood ratio of negative test result 0.10 .

The area under ROC curve was 0.758 (95 $\%$ confidence interval -0.601 to 0.876 ) for IPP and 0.718 (95\% confidence interval -0.558 to 0.846 ) for prostate volume, Figure-2.

A flow diagram for IPP on diagnostic accuracy is showed in Figure-3.

Table 2 - Prostate volume, IPP and BOOI (Kruskal-Wallis; Dunn's test).

\begin{tabular}{lcllllll}
\hline \multicolumn{4}{c}{ Prostate Volume $(\mathrm{mL})$} & \multicolumn{5}{c}{ IPP $(\mathrm{mm})$} \\
\hline BOOI & $\mathrm{N}$ & Mean & SD & BOOI & N & Mean & SD \\
Nonobstructed & 10 & 29.8 & 19.4 & Nonobstructed & 10 & 7.6 & 8.5 \\
Doubt & 12 & 43.2 & 33.1 & Doubt & 12 & 8.5 & 7.0 \\
Obstructed & 20 & 53.6 & 32.9 & Obstructed & 20 & 15.4 & 6.6 \\
\hline
\end{tabular}

$p=0.033$ (prostate volume); $p=0.016$ (IPP); IPP = intravesical prostatic protrusion. 
Table 3 - IPP grade III accuracy.

\begin{tabular}{lccc}
\hline Measurement & \% & $\mathbf{9 5 \%}$ CI & N / Total \\
\hline Sensitivity & 80.0 & $55.7 ; 93.4$ & $19 / 21$ \\
Specificity & 68.2 & $45.1 ; 85.3$ & $19 / 25$ \\
Positive predictive value & 69.6 & $46.9 ; 85.9$ & $13 / 19$ \\
Negative predictive value & 78.9 & $53.9 ; 93.0$ & $19 / 27$ \\
Accuracy & 73.8 & $57.7 ; 85.6$ & $32 / 46$ \\
LR + & 2.51 & & \\
LR - & 0.29 & & \\
\hline
\end{tabular}

$C I=$ confidence interval; IPP = intravesical prostatic protrusion; $L R=$ likelihood ratio; $N=$ number of patients.

\section{COMMENTS}

LUTS are one of the most common complaints in the elderly men and benign prostate obstruction is one of the most frequent causes. Pressure flow study has been recommended before surgical treatment of prostate enlargement by many authors. Searching for new accurate methods that could substitute the gold standard pressure-flow study demonstrates the need for lowering costs, expanding accessibility and relieving patient discomfort .

Since transrectal methods can producegreat discomfort to the patient, abdominal ultrasound was demonstrated to be equivalent to rectal ultrasound for

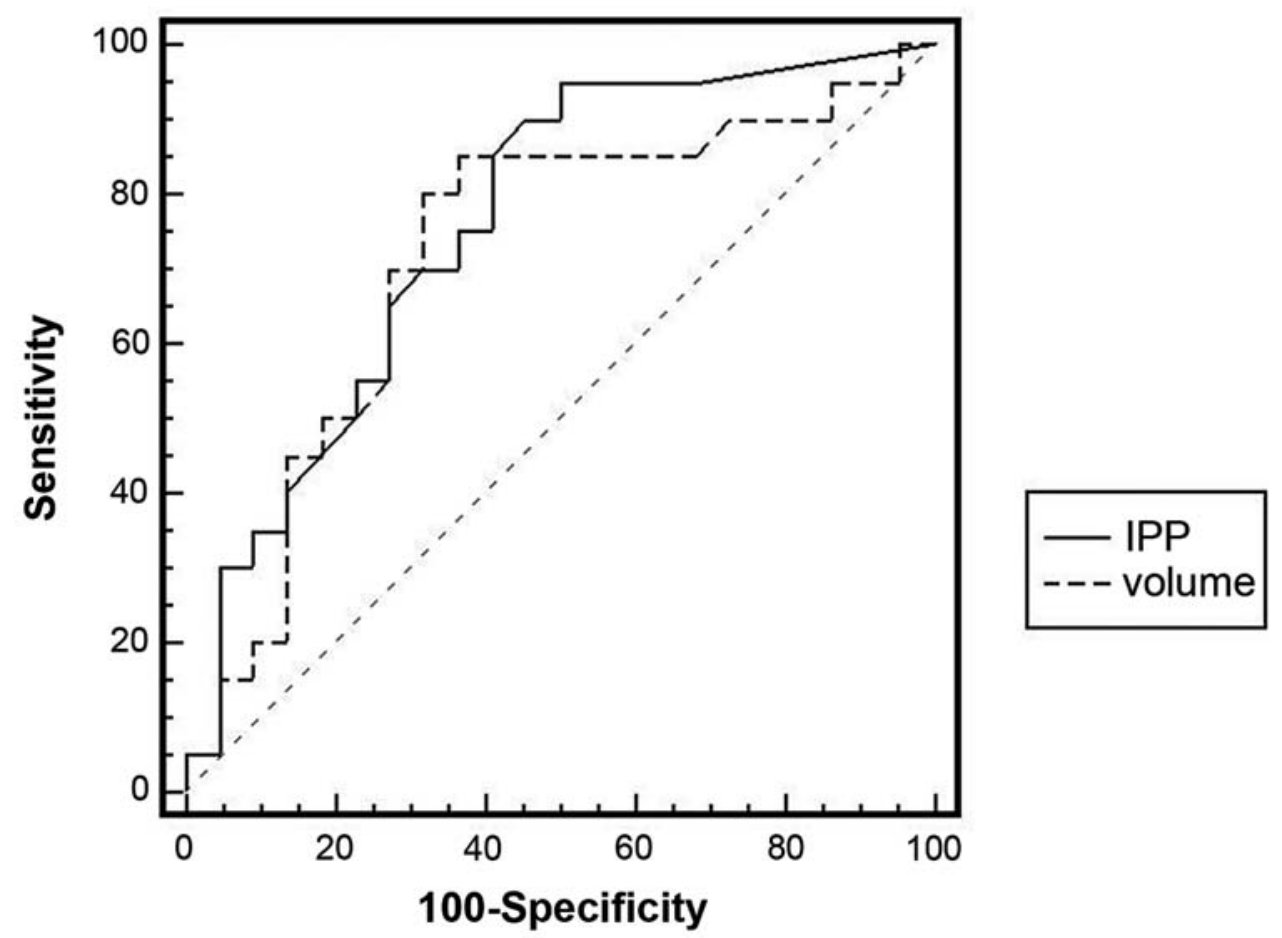

Figure 2-Receiver Operating Characteristic (ROC) curve for intravesical prostatic protrusion (IPP) and prostate volume. 
measuring the prostate when bladder volume is over $100 \mathrm{~mL}(5,6)$.

Clinical data such as IPSS, post voiding residue and flowmetry have been previously demonstrated to correlate mostly to lower urinary tract functional status rather than mechanical obstruction itself (7-9). Therefore, noninvasive measurements of the prostate intend to delineate a morpho-functional correlation in order to orient conduct towards LUTS secondary to benign prostate obstruction.

Almost all studies on IPP measurements come from Asia and it is unknown if the results in Asians are valid for Latin-Americans or Caucasians as well.

Chia et al. (2) demonstrated the possibility of using the IPP measurements for diagnosing BOO, which was also a predictor of the capacity for spontaneous voiding after acute urinary retention in Tan et al. study (10).

Other authors have suggested determining bladder weight, bladder wall width or prostate conformation through abdominal or rectal ultrasound (11-14).

Kojima et al. demonstrated, studying 104 patients, that the bladder weight more than $35 \mathrm{~g}$ performed thought transabdominal ultrasound is strongly associated with bladder outlet obstruction on pressure-flow studies (15).

A bladder wall thickness of $5 \mathrm{~mm}$ appeared to be the best cutoff point to diagnose bladder outlet obstruction, since $63.3 \%$ of patients with bladder wall thickness less than $5 \mathrm{~mm}$ were unobstructed while $87.5 \%$ of those with a bladder wall thickness $5 \mathrm{~mm}$ or greater were obstructed in a study including 174 patients of Manieri et al. at $150 \mathrm{~mL}$ bladder filling (16).

Hakenberg et al. (17) found that mean bladder wall thickness was $3.33 \mathrm{~mm}$ in healthy men and $3.67 \mathrm{~mm}$ in men with LUTS and BPE, measuring all patients at different bladder fillings. BOO was found in $95.5 \%$ of men with a detrusor wall thickness greater than or equal to $2 \mathrm{~mm}$ in Oelke et al. study, at $250 \mathrm{~mL}$ or more bladder filling (18).

Recently, Blatt et al. (19) who performed urodynamics evaluation and abdominal ultrasound among patients with different types bladder dysfunction, found that mean bladder wall thickness in patients with normal urodynamics, bladder outlet obstruction, detrusor overactivity and increased bladder sensation was 2.0, 2.1, 1.9 and $1.8 \mathrm{~mm}$, respectively. No significant difference was found between the groups. In particular, there was no difference in bladder wall thickness between patients with normal urodynamics, and those with bladder outlet obstruction $(\mathrm{p}=0.31)$ or detrusor overactivity $(\mathrm{p}=0.31)$.

The inconsistency as regards the results obtained and the lack of technique standardization have limited their clinical use until now.

Intravesical protrusion seems to corroborate with urinary obstruction through a "valve ball" mechanism, in which the prostate's lateral and medium lobes interfere on the complete opening of the vesical neck while the patient urinates (10). According to this mechanism and based on the present study, it was demonstrated that the intravesical protrusion of the prostate relates not only to the urinary obstruction itself, but it also provides information concerning the severity of obstruction. It has been demonstrated that the greater the IPP, the higher BOOI (20). Still significant, but to a lesser extent, results of prostatic volume obtained through ultrasound and PSA also related to the degree of obstruction (21-24).

Utilizing receiver-operator characteristic curves, the area under the curve for IPP were 0.772, and 0.858 for Lim et al. (21) and Keqin et al. (20), respectively. The latter authors found $8.5 \mathrm{~mm}$ as the best cutoff value for IPP with $75.5 \%$ of sensitivity and 82.6 of specificity.

Our findings, 0.758 for the area under the curve and $5 \mathrm{~mm}$ as the best cutoff value for IPP, are in agreement with these earlier investigations.

On the other hand, our study had a limited number of patients and presented great variability of results, which weakened its immediate clinical application. However, these early statistically significant results lead towards new tendencies and studies necessary to seek improved methods of diagnosing $\mathrm{BOO}$ as well as technique standardization.

\section{CONCLUSION}

IPP and prostatic volume measured through abdominal ultrasound are noninvasive and accessible methods that significantly correlate with urinary BOO, 


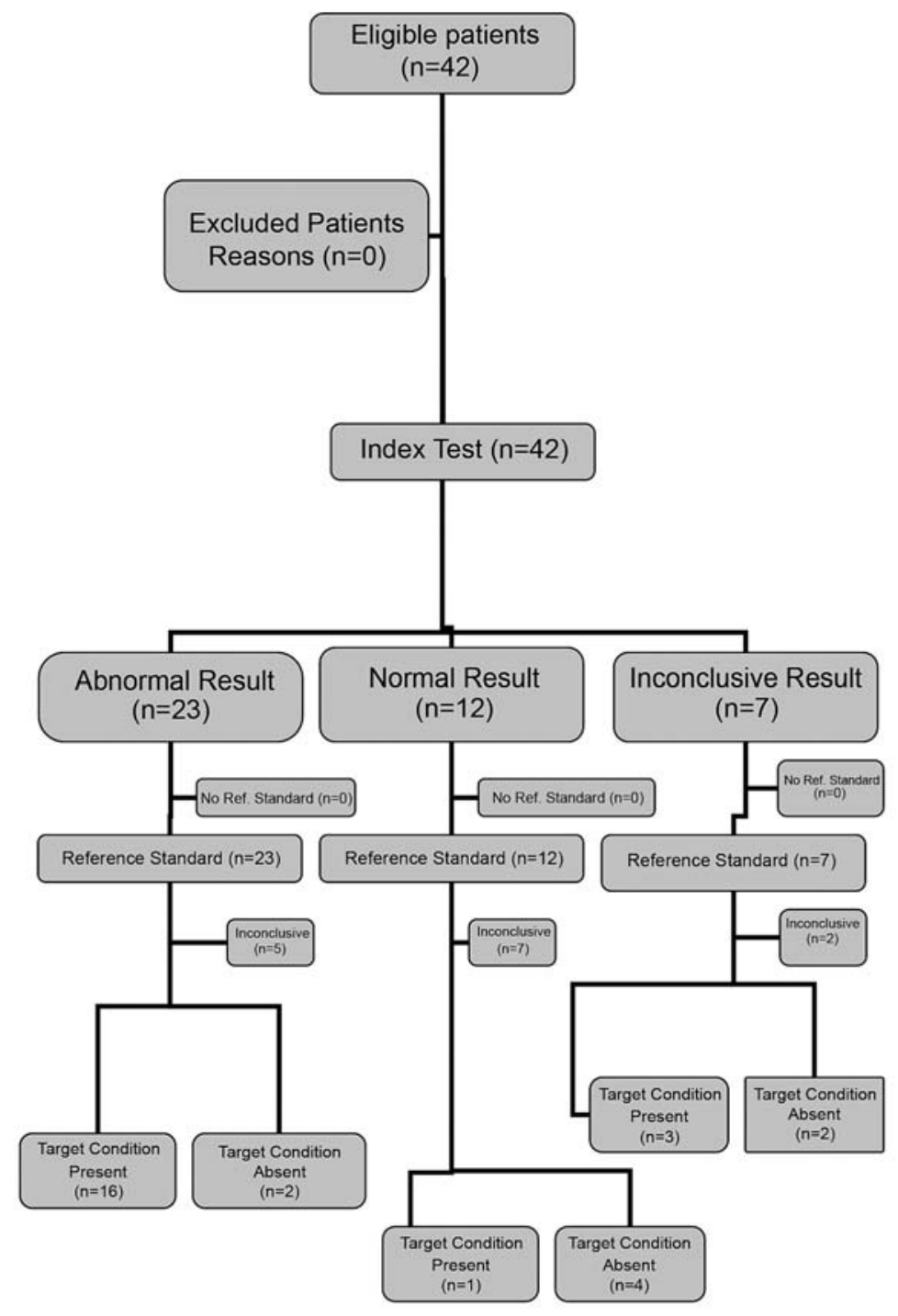

Figure 3 - Flow diagram for intravesical prostatic protrusion on diagnostic accuracy.

and diagnose male urinary obstructive problems. However, results are still variable and the small number of patients in this study renders further studies necessary for a final definite conclusion.

\section{CONFLICT OF INTEREST}

None declared.

\section{REFERENCES}

1. Belal M, Abrams P: Noninvasive methods of diagnosing bladder outlet obstruction in men. Part 1: Nonurodynamic approach. J Urol. 2006; 176: 22-8.

2. Chia SJ, Heng CT, Chan SP, Foo KT: Correlation of intravesical prostatic protrusion with bladder outlet obstruction. BJU Int. 2003; 91: 371-4.

3. Schäfer W, Abrams P, Liao L, Mattiasson A, Pesce F, Spangberg A, et al.: Good urodynamic practices: 
uroflowmetry, filling cystometry, and pressure-flow studies. Neurourol Urodyn. 2002; 21: 261-74.

4. Abrams P: Bladder outlet obstruction index, bladder contractility index and bladder voiding efficiency: three simple indices to define bladder voiding function. BJU Int. 1999; 84: 14-5.

5. Yuen JS, Ngiap JT, Cheng CW, Foo KT: Effects of bladder volume on transabdominal ultrasound measurements of intravesical prostatic protrusion and volume. Int J Urol. 2002; 9: 225-9.

6. Ohnuki T, Kurokawa K, Katoh N, Fukabori Y, Shimizu K, Nakai K, et al.: Transrectal longitudinal ultrasonography of the prostate by electronic linear scanning. Hinyokika Kiyo. 1987; 33: 1385-8.

7. Netto Júnior NR, D’Ancona CA, de Lima ML: Correlation between the International Prostatic Symptom Score and a pressure-flow study in the evaluation of symptomatic benign prostatic hyperplasia. J Urol. 1996; 155: 200-2.

8. el Din KE, Kiemeney LA, de Wildt MJ, Rosier PF, Debruyne FM, de la Rosette JJ: The correlation between bladder outlet obstruction and lower urinary tract symptoms as measured by the international prostate symptom score. J Urol. 1996; 156: 1020-5.

9. Ezz el Din K, Kiemene LA, de Wildt MJ, Debruyne FM, de la Rosette JJ: Correlation between uroflowmetry, prostate volume, postvoid residue, and lower urinary tract symptoms as measured by the International Prostate Symptom Score. Urology. 1996; 48: 393-7.

10. Tan YH, Foo KT: Intravesical prostatic protrusion predicts the outcome of a trial without catheter following acute urine retention. J Urol. 2003; 170: 2339-41.

11. Ochiai A, Kojima M: Correlation of ultrasound-estimated bladder weight with ultrasound appearance of the prostate and postvoid residual urine in men with lower urinary tract symptoms. Urology. 1998; 51: 722-9.

12. Kuo HC: Clinical prostate score for diagnosis of bladder outlet obstruction by prostate measurements and uroflowmetry. Urology. 1999; 54: 90-6.

13. Ockrim JL, Laniado ME, Patel A, Tubaro A, St Clair Carter S: A probability based system for combining simple office parameters as a predictor of bladder outflow obstruction. J Urol. 2001; 166: 2221-5.

14. Kessler TM, Gerber R, Burkhard FC, Studer UE, Danuser $\mathrm{H}$ : Ultrasound assessment of detrusor thickness in mencan it predict bladder outlet obstruction and replace pressure flow study? J Urol. 2006; 175: 2170-3.

15. Kojima M, Inui E, Ochiai A, Naya Y, Ukimura O, Watanabe $\mathrm{H}$ : Ultrasonic estimation of bladder weight as a measure of bladder hypertrophy in men with infravesical obstruction: a preliminary report. Urology. 1996; 47: 942-7.
16. Manieri C, Carter SS, Romano G, Trucchi A, Valenti M, Tubaro A: The diagnosis of bladder outlet obstruction in men by ultrasound measurement of bladder wall thickness. J Urol. 1998; 159: 761-5.

17. Hakenberg OW, Linne C, Manseck A, Wirth MP: Bladder wall thickness in normal adults and men with mild lower urinary tract symptoms and benign prostatic enlargement. Neurourol Urodyn. 2000; 19: 585-93.

18. Oelke M, Höfner K, Wiese B, Grünewald V, Jonas U: Increase in detrusor wall thickness indicates bladder outlet obstruction (BOO) in men. World J Urol. 2002; 19: 443-52.

19. Blatt AH, Titus J, Chan L: Ultrasound measurement of bladder wall thickness in the assessment of voiding dysfunction. J Urol. 2008; 179: 2275-8; discussion 2278-9.

20. Keqin Z, Zhishun X, Jing Z, Haixin W, Dongqing Z, Benkang S: Clinical significance of intravesical prostatic protrusion in patients with benign prostatic enlargement. Urology. 2007; 70: 1096-9.

21. Lim KB, Ho H, Foo KT, Wong MY, Fook-Chong $\mathrm{S}$ : Comparison of intravesical prostatic protrusion, prostate volume and serum prostatic-specific antigen in the evaluation of bladder outlet obstruction. Int $\mathrm{J}$ Urol. 2006; 13: 1509-13.

22. Dicuio M, Pomara G, Vesely S, Morelli G, Fabris FM, Ales $\mathrm{V}$, et al.: The use of prostatic intravesical protrusion correlated with uroflowmetry: a new method to measure obstruction in patients with LUTS due to BOO without using P/F studies. Arch Ital Urol Androl. 2005; 77: 50-3.

23. Nose H, Foo KT, Lim KB, Yokoyama T, Ozawa H, Kumon H: Accuracy of two noninvasive methods of diagnosing bladder outlet obstruction using ultrasonography: intravesical prostatic protrusion and velocityflow video urodynamics. Urology. 2005; 65: 493-7.

24. Oelke M, Höfner K, Jonas U, de la Rosette JJ, Ubbink DT, Wijkstra H: Diagnostic accuracy of noninvasive tests to evaluate bladder outlet obstruction in men: detrusor wall thickness, uroflowmetry, postvoid residual urine, and prostate volume. Eur Urol. 2007; 52: 827-34.

Accepted after revision: August 4, 2008 


\section{EDITORIAL COMMENT}

Benign prostatic hyperplasia (BPH) belongs to the most common benign diseases in the aging men. The prevalence of histological $\mathrm{BPH}$ increases with age and appears in approximately $40 \%$ of men aged 51-60 years and in approximately $90 \%$ of men aged $81-90$ years (1). With increasing life expectancy worldwide more men will have these histological changes in their prostate and the probability of seeking professional help will increase as well. It is doubtful if health care systems can support the financial burden associated with the assessment and treatment of BPH-related symptoms and conditions in the future. Therefore, every approach to make the assessment and treatment easier, faster, and cheaper is highly welcome. The authors of the appending article report about their attempt and introduce a new non-invasive test to a broader public (2).

The BPH disease is characterized by benign prostatic enlargement (BPE), bladder outlet obstruction (BOO), and lower urinary tract symptoms (LUTS). However, no clear correlations have been found between these three components and, therefore, each component has to be evaluated separately. Evaluation of BPE, by digito-rectal examination or transrectal ultrasound measurement, or LUTS, by history or questionnaires, is quick, cheap, easy, and without relevant morbidity. However, assessment of BOO has been more difficult until now. Only pressureflow studies were able to detect and quantify bladder outflow resistance adequately. Urodynamic investigations are invasive, expensive, time-consuming, uncomfortable for the patients, widely unavailable, and necessitate a certain degree of education in terms of performance and interpretation of measurement results and artifacts. The morbidity of urodynamic measurements in men is in the range of $19 \%$ and includes dysuria, urinary tract infection, fever, bleeding, and acute urinary retention (3). There are even patients who died after urodynamic investigations because of urosepsis due to contaminated catheters (4). All of these factors are responsible that pressure-flow studies are only randomly performed. Therefore, there is a strong need to develop alternative techniques to measure $\mathrm{BOO}$ and to overcome the disadvantages of pressure-flow studies.
The article by Leonardo Reis and colleagues provides further evidence that ultrasound measurement of intravesical prostatic protrusion (IPP) is able to detect $\mathrm{BOO}$ in $\mathrm{BPH}$ patients quickly and non-invasively (2). Ultrasound machines belong to the standard armamentarium of urologists and are widely available. The IPP technique is easily applicable and the simple measurement of the distance between the bladder neck and the tip of the prostatic median lobe can qualify the patient as obstructed. IPP measurements were originally developed in Asia and results have also been limited to Asian patients (5). The authors of the current study investigated Latin-Americans with this new technique for the first time and could confirm that an IPP of $10 \mathrm{~mm}$ or more is a sensitive tool to detect BOO in patients from another part of the world (sensitivity $80 \%$, positive predictive value $70 \%$, likelihood ratio of positive test result 2.51 ). Therefore, no ethnical difference seems to exist and IPP measurements are of general value. The authors have to be congratulated to have presented a study, which was conducted according to all quality criteria of diagnostic accuracy tests.

Despite the achievements of the authors, the present study has to be classified as a pilot study to demonstrate the proof of principle. Only 42 patients were included in the trial which seems to be underpowered to draw general conclusions. The results are limited to $\mathrm{BPH}$ patients and other types of $\mathrm{BOO}$ cannot be studied with this technique (e.g. bladder neck stenosis, urethral strictures, or meatus stenosis). Furthermore, specificity of IPP measurements is low $(68 \%)$ and, therefore, patients with an IPP distance of less than $10 \mathrm{~mm}$ cannot be safely classified as unobstructed. Until now, only ultrasound measurements of detrusor or bladder wall thickness have shown to have a high sensitivity (83\%), specificity (95\%), and likelihood ratio of a positive test result (17.6) which are superior to all other classic non-invasive tests for $\mathrm{BOO}$ evaluation (uroflowmetry, measurement of postvoid residual urine or prostate volume) (6). Future studies with adequate power, a multicenter and prospective evaluation approach, and the comparison of IPP with other non-invasive tests are necessary to judge the value of this emerging technique correctly. 


\section{REFERENCES}

1. Berry SJ, Coffey DS, Walsh PC, Ewing LL: The development of human benign prostatic hyperplasia with age. J Urol. 1984; 132: 474-9.

2. Reis LO, Barreiro GC, Baracat J, Prudente A, Levi D'Ancona CA: Intravesical protrusion of the prostate as a predictive method of bladder outlet obstruction. Int Braz J Urol 2008;

3. Klingler HC, Madersbacher S, Djavan B, Schatzl G, Marberger M, Schmidbauer CP: Morbidity of the evaluation of the lower urinary tract with transurethral multichannel pressure-flow studies. J Urol. 1998; 159: 191-4.
4. Cann KJ, Johnstone D, Skene AI: An outbreak of Serratia marcescens infection following urodynamic studies. J Hosp Infect. 1987; 9: 291-3.

5. Chia SJ, Heng CT, Chan SP, Foo KT: Correlation of intravesical prostatic protrusion with bladder outlet obstruction. BJU Int. 2003; 91: 371-4.

6. Oelke M, Höfner K, Jonas U, de la Rosette JJ, Ubbink DT, Wijkstra H: Diagnostic accuracy of noninvasive tests to evaluate bladder outlet obstruction in men: detrusor wall thickness, uroflowmetry, postvoid residual urine, and prostate volume. Eur Urol. 2007; 52: 827-34.

Dr. Matthias Oelke Department of Urology Hanover Medical School Hanover, Germany E-mail: oelke.matthias@mh-hannover.de

\section{EDITORIAL COMMENT}

The concept of measuring intra-vesical protrusion of the prostate by ultrasound as a surrogate for bladder outflow obstruction is not a new one (1). The current paper provides further confirmation of the utility of this measurement and suggests that protrusion of $10 \mathrm{~mm}$ or greater correlates well with urodynamic obstruction (2). Along with other ultrasound-derived measurements such as post-void residual, bladder weight, bladder wall thickness, detrusor resistive index, prostatic weight, appearance, and velocity-flow video-urodynamics, this measurement was developed to prevent the need for, and the morbidity of, multi-channel pressure-flow studies (3). The plethora of different techniques suggests that none is perfect and in fact, in individual patients, cannot yet replace 'invasive' testing. Also, the true morbidity of these studies may not be all that significant (4).

On balance, while being suggestive of bladder outflow obstruction, measuring intra-vesical protrusion of the prostate by either abdominal or trans-rectal ultrasound is likely to remain an interesting but inconclusive finding!

\section{REFERENCES}

1. Ohnishi K, Watanabe H, Ohe H, Saitoh M. Development and clinical significance of protrusion of hypertrophic prostate into the bladder observed by transrectal ultrasonotomography. Nippon Hinyokika Gakkai Zasshi. 1985; 76: 1194-200.

2. Reis LO, Barreiro GC, Baracat J, Prudente A, Levi D'Ancona CA: Intravesical protrusion of the prostate as a predicting method of bladder outlet obstruction. Int Braz J Urol. 2008; in press.

3. Klingler HC, Madersbacher S, Djavan B, Schatzl G, Marberger M, Schmidbauer CP: Morbidity of the evaluation of the lower urinary tract with transurethral multichannel pressure-flow studies. J Urol. 1998; 159: 191-4.

4. Porru D, Madeddu G, Campus G, Montisci I, Scarpa RM, Usai E: Evaluation of morbidity of multi-channel pressureflow studies. Neurourol Urodyn. 1999; 18: 647-52.

Dr. Peter Gilling Department of Urology Tauranga Hospital Tauranga, New Zealand E-mail:Peter@promed.co.nz 


\section{EDITORIAL COMMENT}

Benign prostatic hyperplasia (BPH) is one of the most common diseases in elderly men. Bladder outlet obstruction (BOO) should be well discriminated from $\mathrm{BPH}$ to better understand the pathology-physiology of this disease. BPH may lead to benign prostatic enlargement (BPE), BOO and lower urinary tract symptoms (LUTS). BOO might be or not be present in patients with $\mathrm{BPH}$. On the other hand $\mathrm{BOO}$ may cause secondary bladder dysfunction and furthermore upper urinary tract damage. For these reasons, patients with BPH must be evaluated not only for LUTS but also for BOO.

Up to now, urodynamic evaluations have been accepted as the only objective method of assessing BOO. There is a lot to say about the disadvantages of pressure flow studies (PFS) which has been well described in the literature. It is invasive, uncomfortable, time-consuming and expensive. Moreover, there is a need for urethral catheterization, which causes partial obstruction during micturition and confers the undesirable consequences of possibly introducing infection and discomfort that may alter the micturition reflex. Hematuria, urinary tract infection and difficulty in urination are the side effects of this procedure (1). To avoid these disadvantages, in the last decade, the development of non-invasive evaluations for BOO has been the subject of numerous publications. Uroflowmetry, post-void residual urine, prostate volume (PV), bladder wall thickness and finally measurement of intravesical protrusion of the prostate (IPP) are used to estimate $\mathrm{BOO}$ in men with BPH. The rise of the idea that IPP might be a predictor of BOO can be explained by few words. IPP is caused by the enlarging lateral lobes and the median lobe, and may lead to dyskinetic movement of the bladder during voiding. This would cause more obstruction than if there were no protrusion and just bilateral lateral lobes, as the strong bladder contraction could force open a channel between the lobes.

A few investigators have considered IPP to be a useful predictor for evaluating $\mathrm{BOO}$ and bladder function. Chia et al. have suggested that IPP significantly correlates with $\mathrm{BOO}$ and is a better parameter than the other non-invasive parameters (2). Lim et al. have confirmed this study by comparing PV, prostate specific antigen (PSA) and IPP in the evaluation of $\mathrm{BOO}$ and IPP was the strongest predictor in this prospective study (3). In another study, it has been suggested that IPP degree is negatively correlated with Qmax and of patients with higher IPP degree, there is a higher presence of bladder overactivity and low bladder compliance.

Reis et al. provide a prospective data aiming to demonstrate whether the IPP of the prostate might replace the urodynamic evaluation, which is accepted to be an invasive and uncomfortable procedure (4). Despite the small number of patients, the results are in favor of detecting IPP might be enough to demonstrate the $\mathrm{BOO}$ without the need to urodynamic evaluation and comparable with the earlier investigations. These statistically significant results may lead to further investigations and force the urologists to replace measuring IPP instead of performing pressure flow studies in selected patients.

\section{REFERENCES}

1. Klingler HC, Madersbacher S, Djavan B, Schatzl G, Marberger M, Schmidbauer CP: Morbidity of the evaluation of the lower urinary tract with transurethral multichannel pressure-flow studies. J Urol. 1998; 159: 191-4.

2. Chia SJ, Heng CT, Chan SP, Foo KT: Correlation of intravesical prostatic protrusion with bladder outlet obstruction. BJU Int. 2003; 91: 371-4.

3. Lim KB, Ho H, Foo KT, Wong MY, Fook-Chong $\mathrm{S}$ : Comparison of intravesical prostatic protrusion, prostate volume and serum prostatic-specific antigen in the evaluation of bladder outlet obstruction. Int $\mathrm{J}$ Urol. 2006; 13: 1509-13.

4. Reis LO, Barreiro GC, Baracat J, Prudente A, D'Ancona CA: Intravesical protrusion of the prostate as a predictive method of bladder outlet obstruction. Int Braz J Urol. 2008; in press.

Dr. Mesrur Selcuk Silay 2nd Department of Urology Sisli Etfal Training and Research Hospital Istanbul, Turkey E-mail: selcuksilay@gmail.com 


\section{REPLY BY THE AUTHORS}

Although the current and others up-tothe-minute papers provide further confirmation of the utility of intravesical prostatic protusion (IPP) measurements and suggests that protrusion of $10 \mathrm{~mm}$ or greater correlates well with urodynamic obstruction, it is a limited method to accurately define obstruction (1). There are patients obstructed without IPP and unobstructed ones presenting with more than $10 \mathrm{~mm}$ IPP. By the other side, IPP measurements are still a science under development, and perhaps IPP measurements are more precise in determining the best patients for surgical treatment, once they were proved obstructed.

Intravesical protrusion seems to corroborate with urinary obstruction thought a "valve ball" mechanism in which the prostate's lateral and medium lobes interfere on the complete opening of the vesical neck during voiding. This way, the pharmacological response to alphablockers could be predicted by the IPP method (2). Men with an intravesical prostatic protrusion of $10 \mathrm{~mm}$ or less, compared to those with a larger intravesical prostatic protrusion, were 6 times more likely to have a successful trial without catheter after acute urinary retention (3).

Most patients in our present study presenting IPP of $10 \mathrm{~mm}$ or greater showed no response to alpha blockers and were submitted to surgical treatment in contrast to that presenting IPP of less than $10 \mathrm{~mm}$. We are now conducting new prospective studies to prove the accuracy of this method to predict pharmacological treatment outcomes and surgical treatment suggestions.

Another minimal invasive method utilizes ultrasound measurements of detrusor or bladder wall thickness or weight. This method is inconsistent in technical standardization and there is no consensus among authors about its value (4). Bladder parameter to define obstruction is possibly not the best one, because it denotes the obstruction repercussion and imbalance in the detrusor function, which is much more than obstruction and probably occurs latter in the bladder neck obstruction process.

\section{REFERENCES}

1. Yu HF, He YH, Yu KY, Wang Q, Huang PT, Yang Y, et al.: Transabdominal ultrasound measurement of intravesical prostatic protrusion helps diagnosis of benign prostatic obstruction. Zhonghua Nan Ke Xue. 2008; 14: 628-30.

2. Tan YH, Foo KT: Intravesical prostatic protrusion predicts the outcome of a trial without catheter following acute urine retention. J Urol. 2003; 170: 2339-41.

3. Mariappan P, Brown DJ, McNeill AS: Intravesical prostatic protrusion is better than prostate volume in predicting the outcome of trial without catheter in white men presenting with acute urinary retention: a prospective clinical study. J Urol. 2007; 178: 573-7; discussion 577.

4. Blatt AH, Titus J, Chan L: Ultrasound measurement of bladder wall thickness in the assessment of voiding dysfunction. J Urol. 2008; 179: 2275-79.

\section{The Authors}

